Despite, and perhaps because of, the comparatively small numbers of child psychotherapists in the UK the profession is assiduous in presenting the nature and scope of the work which they undertake. This book provides an overview of the background to the establishment of the profession, its development and the diversity of work for which child psychotherapists are trained. There are chapters describing the potential roles through which they may contribute in different settings using formal psychoanalytic psychotherapy, consultative therapeutic work with children and families and consultation with professionals: detailed clinical descriptions bring the work vividly to life. Hodges provides an important summary of research. It was also with some relief that I found a section grappling with the issue of assessment: this could perhaps have been expanded since in practice there is all too often a leap from concern for a child to an assumption that 'therapy' is needed. Fuller appreciation of the process of assessment can only enhance the proper use of psychotherapeutic resources.

The foreword and introduction give acknowledgement to Boston \& Daws' (1977) forerunner of this book and it is this which crystallises the only disappointment I have in this volume. The Concise Oxford Dictionary defines a 'handbook' as "(usually short) treatise, manual, guidebook". However, Lanyado \& Horne's book is not one I could direct someone towards in the process of a particular treatment in order to gain specific guidance in a structured way: nor does it provide a systematic introductory text for training in psychoanalytic psychotherapy with children. At times there seems to have been the insertion of an obligatory reference to 'the child psychotherapist' when what is at issue is 'psychoanalytic psychotherapy with children': this felt intrusive and I fear could distract into an unhelpful debate about the roles of different professions. I do not feel I can represent this as a handbook but it is an extremely important and valuable development from its forerunner whose title accurately describes it, The Child Psychotherapist and the Problems of Young People.

The style of writing is generally very clear and should prove accessible to those without a foundation in psychoanalytic theory. It will be helpful to practitioners without specific training in psychotherapy across the mental health and child welfare fields: many of the sections will also be useful to more experienced practitioners. It should be available in all child mental health service departments.

Boston, M. \& Daws, D. (eds) (1977) The Child Psychotherapist and the Problems of Young People. London: Wildwood House.

Adrian Sutton Consultant in Child \& Family Psychiatry and Psychotherapy, The Winnicott Centre, 195 Hathersage Road, Manchester MI3 0JE

\section{Psychopharmacology of Antipsychotics}

By Stephen M. Stahl. London: Martin Dunitz. 1999. 148 pp. $€ 12.95$ (pb).

ISBNI-853I7-60I-X

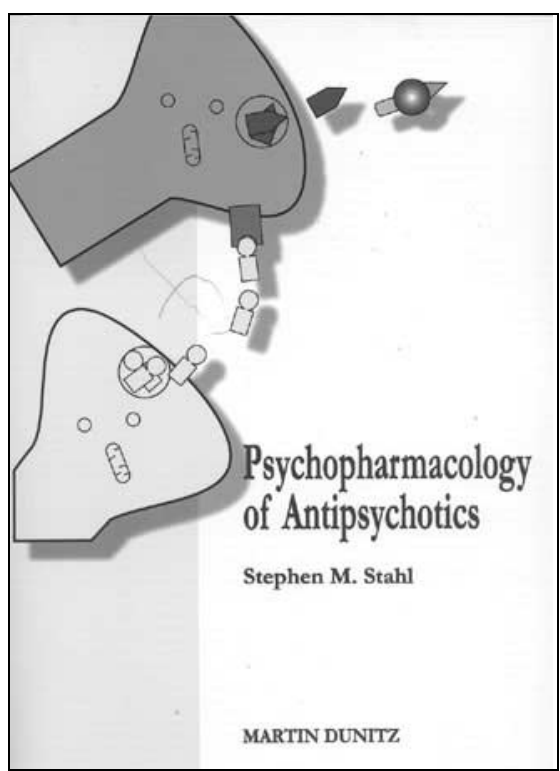

This book is written in a concise and readerfriendly format which makes it difficult to put down. It is colourful, makes excellent use of lists and tables and avoids an overload of information about what can seem a confusing subject to the clinician. One of its strongest points are the illustrations of cells, receptors and neuronal pathways. It is as up-to-date as possible in this rapidly changing subject and proactive in containing information about drugs that are likely to come onto the market soon.

This book is about more than just antipsychotic drugs. The reader is taken on a journey from neurotransmitters and pharmacokinetics to discussions about the relative merits of the new antipsychotics and strategies for the drug treatments of the different syndromes of schizophrenia. I was especially interested in the lucid depiction of the neurodevelopmental and neurodegenerative hypotheses of the aetiology of schizophrenia. The chapter introduces the role of the glutamate system and speculates about a role for neuroprotective agents. There are tables of 'clinical pearls' and 'dosage tips' which provide useful information about each drug.

My criticisms of this book are relatively minor. Its main drawback is that although it takes an international perspective, it omits any information about the drugs sulpiride and amisulpride which have never been marketed in the USA. They are considered by some authors to have atypical properties. Amisulpride has been shown to improve negative symptoms of schizophrenia in well-designed trials, particularly selected for patients with primary negative symptoms. The discussion about drug treatment of negative symptoms therefore seems incomplete.

The discussions of drug costs need to be interpreted with caution. For example, the author states the different dose tablets $(2.5 \mathrm{mg}, 5 \mathrm{mg}, 7.5 \mathrm{mg}$ and $10 \mathrm{mg})$ of olanzapine "can cost the same" price. This is not confirmed by reference to the British National Formulary or the pharmacy at my hospital, although costs may vary between services. As a practising clinician, reference to the difficult situations for prescription of antipsychotics, such as pregnancy and after neuroleptic malignant syndrome, would have also been helpful. Finally, the author contrasts the data from drug trials with clinical experience in the final chapter which summarises drug strategies for schizophrenia. However, references appear in only limited parts of the text. Statements such as "optimal doses derived from clinical trials do not match optimal doses used in clinical practice" and "atypical antipsychotics do not seem to work as fast as conventional antipsychotics" left me urgently wanting to read further to access the source of this potentially important information.

These minor criticisms excepted, it will allow psychiatrists, general practitioners and allied mental health professionals to keep up to date with the latest developments in this rapidly evolving subject. It will inspire psychiatric trainees in their revision for the MRCPsych Part I psychopharmacology and Part II neurochemistry sections of the syllabus. I regret that such a 
textbook was not available at the time I took my exams.

Robert Chaplin Consultant Psychiatrist, South West London and St George's Mental Health Trust, London SWI7 7DJ

\section{Atlas of Psychiatric Pharmacotherapy}

By R. Shiloh, D. Nutt \& A.Weizman. London: Martin Dunitz. 1999. 235 pp. $€ 49.95$ (hb). ISBN I-85317-630-3

Some people (usually males) have superior visuo-spatial abilities. Such men excel at map-reading, a skill at which women are allegedly abysmal. Those of either gender who delight in maps will enjoy this book. Those with predominantly linguistic tendencies may feel like a hippocampamised rat lost in a maze.

The book, by title and aim, is an atlas "written ... for the clinician required to know, understand, and decide efficiently about options for biological treatments". It contains a series of intracellular road maps depicting not only the main highways travelled by psychotropic drugs but also the smaller roads and footpaths, some of which peter out, ending in "unknown cellular mechanisms". These maps are ingenious, intricate but difficult. They follow the route, for example, of an antidepressant drug from a serotonin receptor through many stages to its effect on "cAMP response element binding protein (CREB) and the 'proper production' of 'brain-derived neurotropic factor (BDNF), ", leading finally to resolution of the depressive state. It is doubtful whether many psychiatrists will penetrate this far into the jungle. However, the maps, with their densely written texts, could be valuable for research workers requiring detailed information, and may serve as a reference for clinicians to absorb at leisure. Students and trainees would do better to start with something simpler.

Other maps, more easily followed, include flow charts of treatment strategies and grids of adverse drug effects. The former are available from other sources, but this book has the merit of stating how firmly evidence-based are the strategies suggested. Nevertheless, the text requires close searching for important clinical information such as dosage titration, dosage in the elderly and individual metabolic differences. Withdrawal reactions from antidepressants or antipsychotics are not mentioned among adverse effects, although the flow charts advise slow tapering of dosage on withdrawal (no reason given). Readers may disagree with various items such as the statements that withdrawal reactions occur "only if stopped quickly" for several short half-life benzodiazepines and are "relatively insignificant" with zopiclone.

These are perhaps minor criticisms considering the wealth of information the book contains, and its wide coverage spanning basic pharmacodynamics and pharmacokinetics, drug interactions, drugs of misuse, sexual dysfunction, personality and eating disorders and attention-deficit hyperactivity disorder. The authors deserve congratulations for their originality and courage in presenting in black and white (and colour) their micro- and macroscopic perspectives on an extremely complicated subject. This book is a must for the library.

C. Heather Ashton School of Neurosciences, Division of Psychiatry, University of Newcastle, The Royal Victoria Infirmary, Queen Victoria Road, Newcastle uponTyne NEI 4LP

\section{Prenatal Exposures in Schizophrenia}

Edited by Ezra S. Susser, Alan S. Brown \& Jack M. Gorman. Washington, DC: American Psychiatric Press.1999.261 pp.US\$36.50 (hb). ISBN 0-88048-499-3

If you attend an annual meeting of the American Psychiatric Association (APA), you are faced every day with a bewildering array of symposia, plenary sessions, workshops, posters and so on. More often than not you make the wrong choice and that evening at some social function you are told about the session you 'really should have attended'. The APA must be aware of this because it now produces the 'Progress in Psychiatry Series' which publishes 'the best of the symposia'. This book is number 56 - clearly the APA is on to a good thing (Royal College please note).

Although it is not clear in the introduction, I presume this symposium was organised by Dr Ezra Susser of Columbia University in New York. There are only nine chapters, but six come from his department. The book is really an extension of his already published work, which argues forcefully for the role of prenatal factors in the aetiology of schizophrenia.
Nowakowski from New Jersey sets the scene with a useful description of normal prenatal brain development (but why is this Chapter 3?). Waddington et al from Ireland review especially their own work that suggests a 'cascade process' in which intrauterine events are associated in childhood with neuromotor and psychological abnormalities and in adulthood with the appearance of psychosis. But the core of the book is a description and expansion of Susser et al's finding that in the Dutch famine at the end of the Second World War, those who were exposed as foetuses in the first trimester of pregnancy to the height of the famine, had a greater than expected chance of developing schizophrenia in adult life. Susser believes that prenatal nutrition may be an important risk factor in schizophrenia; his current work is taking this further by looking at specific micronutrients.

This is an intriguing finding. However, to the best of my knowledge it has not been replicated. There must be many other specific situations in which pregnant mothers are exposed to famine. Also, what about chronic severe malnutrition in a country such as India? If Susser is right, then why is the incidence of schizophrenia not demonstrably higher in that country; or is it that such children die before reaching adulthood?

What I am really trying to say, I suspect, is that in this book there is no chapter providing a counterbalance to the undoubted enthusiasm of Susser's group. If Professor Tim Crow had been invited to speak at the symposium, there might have been a real debate.

For me, interested in clinical research in schizophrenia, this book will provide a useful source of references; I suspect it is not for the general psychiatrist.

Robin G. McCreadie Director of Clinical Research, Crichton Royal Hospital, Dumfries DGI 4TG

\section{Psychiatric and Behavioural Disorders in Developmental Disabilities and Mental Retardation}

Edited by Nick Bouras. 1999. Cambridge:

Cambridge University Press.

464 pp. $€ 35.00$. ISBN 0-52I-64395-3

This excellent postgraduate textbook provides a broad critical appraisal of psychiatric and behaviour disorders in individuals 